\title{
Surface chemical analysis of the effect of extended laundering on C. I. sulphur black 1 dyed cotton fabric
}

\author{
G. Soliman ${ }^{\mathrm{a}, *}$, C.M. Carr ${ }^{\mathrm{a}}$, C.C. Jones ${ }^{\mathrm{b}}$, M. Rigout ${ }^{\mathrm{a}}$ \\ ${ }^{a}$ Textiles, School of Materials, University of Manchester, Manchester M13 9PL, UK \\ ${ }^{\mathrm{b}}$ Unilever Research, Port Sunlight Laboratory, Wirral CH63 3JW, UK
}

\section{A R T I C L E I N F O}

\section{Article history:}

Received 14 May 2012

Accepted 27 June 2012

Available online 14 July 2012

\section{Keywords:}

Cotton

Sulphur dye

Wash fastness

XPS

Laundering

Colour

\begin{abstract}
A B S T R A C T
The colour resulting from the laundering of cotton fabric is influenced by the nature of the dye, the detergent formulation and the physical structure of the fibre surface. In this study the effect of ISO (International Organisation for Standardization) 105 CO6 and ISO 105 CO9 wash fastness testing, with and without oxidant, and with and without stainless steel abrasive balls, on the colour of C.I. Sulphur Black 1 dyed cotton fabric was evaluated. The surface sensitive X-ray Photoelectron Spectroscopy (XPS) was used to probe the nature of the outer $3-10 \mathrm{~nm}$ of the cotton surface, characterise the surface functionalities and elemental composition and establish the durability of the surface dye to different washing regimes. In addition the effect of laundering and modification to the surface sulphur dye was related to colour through $K / S$ and $L^{*}$ analysis.
\end{abstract}

(ㄷ) 2012 Elsevier Ltd. All rights reserved.

\section{Introduction}

Sulphur dyes are one of the most popular colourants used on cellulosic fibres and their blends [1-4]. C. I. Sulphur Black 1 in particular represents a substantial portion (20-25\%) of the dyestuff market for cotton and has been reported to have the highest consumption of any single textile dyestuff in the world [5]. Sulphur dyes are characterised by their moderate cost and acceptable fastness for medium to heavy depths, especially black, blue and brown $[3,4,6]$. In spite of sulphur dyes offering excellent light fastness and good wash fastness to non-perborate based detergents, their commercial application in recent years has declined due to the availability of relatively dull colours, limited colour gamut, relatively poor wet rub fastness, the possibility of overoxidation of the leuco form back to the colourant and the environmental impact associated with the presence of sulphides in the effluent [7,8]. However the environmental impact of sulphur dyeing can be significantly reduced with the use of "greener" reducing agents, such as sugars [3,5,9].

The application of the water insoluble sulphur dyes to cotton involves an initial alkaline reduction to the water soluble leuco

\footnotetext{
* Corresponding author. Tel.: +44 1613064131.

E-mail addresses: ghada.soliman@postgradmanchester.ac.uk, soliman_ghada@ yahoo.com (G. Soliman), chris.carr@manchester.ac.uk (C.M. Carr), christopher.jones@unilever.com (C.C. Jones), muriel.rigout@manchester.ac.uk (M. Rigout).
}

form and the subsequent absorption of the dye into the fibres. After exhaustion the leuco dye is re-oxidised using an oxidant, such as hydrogen peroxide, back to the original water insoluble form within the fibre and any surface dye is removed with after-soaping $[1,2]$. The fastness and cross-staining performance of sulphur dyed cellulosic fibres on exposure to severe washing conditions, especially those that use peroxide-containing detergent, has been typically characterised using the standard ISO 105 C06 benchmark [10-12]. However, recently a more representative ISO 105 CO9 test method, reflecting modern detergent formulations and laundering practice, has been developed using a standard detergent incorporating a low temperature tetraacetylethylenediamine (TAED) bleach activator, incorporating either a single or multiple wash cycles [11-13].

In this study the relative effect of ISO 105 C06 [10] and ISO 105 C09 [13] washing, with and without oxidant and with and without stainless steel abrasive balls, on the colour of C. I. Sulphur Black 1 dyed cotton fabric has been evaluated and their chemical and physical effect on the outer surface of the Sulphur Black dyed fibres investigated for the first time. The surface sensitive X-ray Photoelectron Spectroscopy (XPS) technique $[14,15]$ was used to probe the nature of the outer 3-10 nm of the cotton's surface and in order to characterise the surface functionalities, establish the elemental composition and assess the durability of the surface sulphur dye to different washing regimes. Wet laundering of cellulosic fibres can also produce mechanical damage to the fibre resulting in fibrillation and a loss in colour intensity due to diffuse reflection of light at 
the fibre surface [16-21]. Therefore by fully understanding the nature of the fibre surface interface on exposure to chemically and physically aggressive media improved laundering processes and extended garment lifetime may be achieved.

\section{Experimental}

\subsection{Materials}

A plain woven, bleached, $100 \%$ cotton fabric, $135 \mathrm{~g} / \mathrm{m}^{2}$, supplied by Phoenix Calico Ltd., UK, was used throughout this study and dyed with 5\% o.w.f. (on mass of fabric) C. I. Sulphur Black 1. The ECE non-phosphate detergent, ECE phosphate detergent and tetraacetylethylenediamine (92\% active) were purchased from the Society of Dyers and Colourists (SDC), Bradford, UK. Sodium perborate tetrahydrate (97\% active) was purchased from Aldrich Chemicals Ltd., UK.

\subsection{Methods and procedures}

\subsubsection{Dyeing procedure [20]}

The cotton fabric was introduced into a dyebath containing $50 \mathrm{~g} / \mathrm{L}$ Diresul Black B (C. I. Sulphur Black 1), $25 \mathrm{~g} /$ L sodium chloride, $13 \mathrm{ml} / \mathrm{L}$ sodium hydroxide (67 $\left.{ }^{\circ} \mathrm{Tw}\right), 9 \mathrm{~g} / \mathrm{L}$ Diresul Reducing agent $\mathrm{D}$ and $1 \mathrm{~g} / \mathrm{L}$ of Sandopan CBN at $20{ }^{\circ} \mathrm{C}$ and a liquor:goods ratio of $10: 1$. The dyebath was raised to $98{ }^{\circ} \mathrm{C}$ over $20 \mathrm{~min}$ and maintained at the boil for $60 \mathrm{~min}$. The fabric was then rinsed in cold water and oxidised with $40 \mathrm{~g} / \mathrm{L}$ Sandopur DSC (oxidant and soaping agent) and $20 \mathrm{~g} / \mathrm{L}$ sodium carbonate at $98^{\circ} \mathrm{C}$ for $20 \mathrm{~min}$. The fabric was then soaped in $1 \mathrm{~g} / \mathrm{L}$ non-ionic detergent solution for $5 \mathrm{~min}$ at $60^{\circ} \mathrm{C}$, rinsed in water and dried at room temperature.

\subsubsection{Laundering conditions}

The C. I. Sulphur Black 1 dyed fabric was washed as described in the ISO 105 CO6 C1S/C2S [10] procedures. The wash fastness test involved treating the dyed fabric in a solution containing $4 \mathrm{~g} / \mathrm{L} \mathrm{ECE}$ detergent, with or without $1 \mathrm{~g} / \mathrm{L}$ sodium perborate, with or without 25 stainless steel balls, adjusted to $\mathrm{pH} 10.5$, at $60^{\circ} \mathrm{C}$ for $30 \mathrm{~min}$. The fabric was finally rinsed in running water at $40{ }^{\circ} \mathrm{C}$ and air dried at room temperature.

Dyed fabric was also washed in accordance with the ISO 105 CO9 [13] procedure, again with or without the perborate. The wash fastness test involved introducing the black dyed fabric into a solution containing $10 \mathrm{~g} / \mathrm{L} \mathrm{ECE}$ non-phosphate detergent, $1.8 \mathrm{~g} / \mathrm{L}$ TAED, with or without $12 \mathrm{~g} / \mathrm{L}$ sodium perborate, with or without 25 stainless steel balls, at $25^{\circ} \mathrm{C}$ and raising the temperature to $60^{\circ} \mathrm{C}$ at $2{ }^{\circ} \mathrm{C} / \mathrm{min}$ and maintaining at $60^{\circ} \mathrm{C}$ for $30 \mathrm{~min}$. The fabric was finally rinsed in running water at ambient temperature and air dried at room temperature prior to further analysis.

\subsubsection{Colour analysis}

The colour strength $(K / S)$ was calculated from reflectance measurements using the Kubelka-Munk equation (Equation (1)):

$$
\left(\frac{K}{S}\right)_{\lambda}=\frac{\left(1-R_{\lambda}\right)^{2}}{2 R_{\lambda}}
$$

where $K$ is the absorption coefficient, $S$ is the scatter coefficient, $R$ is reflectance expressed as a fractional value.

$K / S$ and CIE $L^{*} a^{*} b^{*}$ values were calculated using a Datacolor Spectroflash 600 and the mean value was an average of four measurements. The $K / S$ values in Table 2 were calculated at a $\lambda_{\max }$ of $580 \mathrm{~nm}$.
Table 1

XPS surface elemental composition of ISO 105 CO6/CO9 washed sulphur black dyed cotton fabrics.

\begin{tabular}{lllll}
\hline Washing treatment & \multicolumn{3}{l}{ XPS atomic \% } & \\
\cline { 2 - 5 } & Carbon & Nitrogen & Oxygen & Sulphur \\
\hline None & 73.5 & 2.6 & 21.0 & 3.0 \\
ISO 105 CO6 & & & & \\
With perborate $^{\mathrm{a}}$ & 68.9 & 2.1 & 26.3 & 2.7 \\
With perborate $^{\mathrm{b}}$ & 71.2 & 2.6 & 23.5 & 2.8 \\
Without perborate $^{\mathrm{a}}$ & 72.0 & 2.8 & 21.9 & 3.0 \\
Without perborate $^{\mathrm{b}}$ & 70.0 & 2.4 & 24.9 & 2.7 \\
With perborate $^{\mathrm{a}, \mathrm{c}}$ & 69.3 & 1.7 & 27.3 & 1.6 \\
Without perborate $^{\mathrm{b}, \mathrm{c}}$ & 72.2 & 2.6 & 22.0 & 3.2 \\
ISO 105 CO9 $_{\text {With perborate }}^{\mathrm{a}}$ & 69.0 & 0.7 & 30.0 & 0.6 \\
With perborate $^{\mathrm{b}}$ & 68.3 & 0.4 & 30.5 & 0.8 \\
Without perborate $^{\mathrm{a}}$ & 70.2 & 2.3 & 24.5 & 3.0 \\
Without perborate $^{\mathrm{b}}$ & 71.5 & 2.9 & 22.7 & 2.9 \\
\hline
\end{tabular}

${ }^{\text {a }}$ With stainless steel balls.

b Without stainless steel balls.

c Triplicate washing cycles.

\subsubsection{XPS analysis}

XPS analysis was performed using a Kratos Axis system spectrometer. The fabric samples were cut from the middle of the specimen and attached to the sample holder using a double sided tape. Monochromatic Al $\mathrm{K}_{\alpha}$ X-rays $(1486.6 \mathrm{eV})$ with a power of $150 \mathrm{~W}$ were used to irradiate the samples and wide scan spectra were recorded with a pass energy of $160 \mathrm{eV}$ from which the surface composition (C, O, S and N) was determined. High resolution Carbon (1s) and Sulphur (2p) spectra were recorded with pass energy of $40 \mathrm{eV}$ and the binding energy (BE) values were calculated relative to Carbon (1s) photoelectron peak at $285.0 \mathrm{eV}$. Charge compensation for the samples was achieved using a 4-7 eV beam at a flood current of approximately $0.1 \mathrm{~mA}$, with an electrically ground $90 \%$ transmission nickel mesh screen. All samples were analysed in duplicate and data analysed using the CASA XPS software [22].

\subsubsection{SEM analysis}

A Hitachi S3000N variable pressure instrument was used throughout the study. A magnification of 2000:1, a $5 \mathrm{kV}$ accelerating voltage and a working distance of $10 \mathrm{~mm}$ were typically found to be the most appropriate analysis conditions and the samples were gold coated prior to analysis.

Table 2

Effect of ISO 105 CO6/CO9 washing on the colour of sulphur black dyed cotton fabrics.

\begin{tabular}{lll}
\hline Washing treatment & $K / S$ & $L^{*}$ \\
\hline None & 23.2 & 16.1 \\
ISO 105 CO6 & & \\
With perborate $^{\mathrm{a}}$ & 21.1 & 17.1 \\
With perborate $^{\mathrm{b}}$ & 22.8 & 16.4 \\
Without perborate $^{\mathrm{a}}$ & 21.5 & 17.0 \\
Without perborate $^{\mathrm{b}}$ & 22.4 & 16.5 \\
With perborate $^{\mathrm{a}, \mathrm{c}}$ & 19.2 & 18.3 \\
Without perborate $^{\mathrm{b}, \mathrm{c}}$ & 21.3 & 17.0 \\
ISO 105 CO9 $_{\text {With perborate }}^{\mathrm{a}}$ & & \\
With perborate $^{\mathrm{b}}$ & 8.8 & 28.4 \\
Without perborate & 8.4 & 29.0 \\
Without perborate $^{\mathrm{b}}$ & 21.6 & 16.9 \\
\hline
\end{tabular}

\footnotetext{
a With stainless steel balls.

b Without stainless steel balls.

c Triplicate washing cycles.
} 


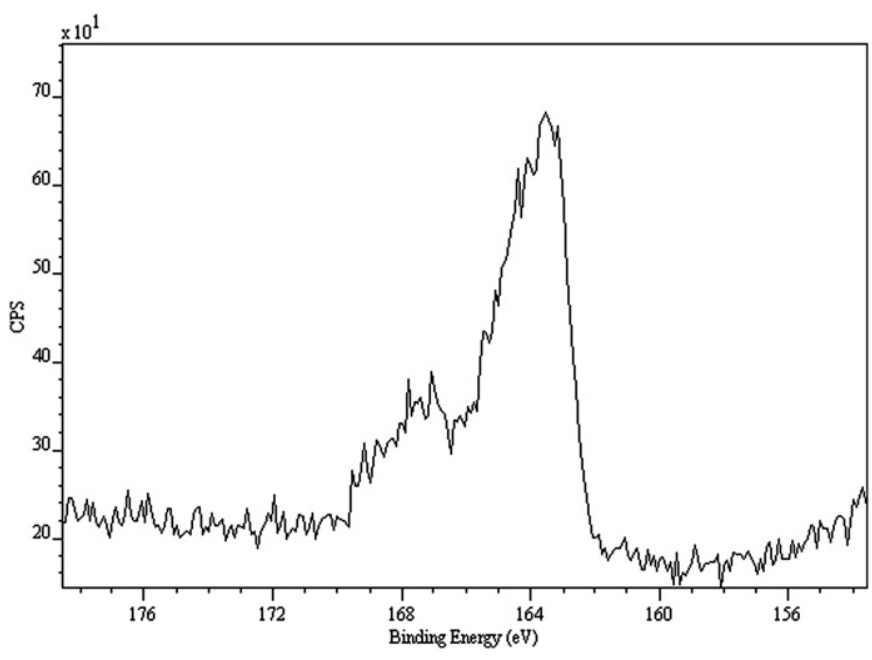

Fig. 1. $S(2 p) X P$ spectrum of unlaundered sulphur black dyed cotton fabric.

\section{Results and discussions}

\subsection{ISO 105 CO6 washing}

Examination of the S(2p) XP spectrum of C. I. Sulphur Black 1 dyed cotton fabric demonstrates the presence of sulphur at the surface of the cotton fibres, with the major peak intensity at $164.0 \mathrm{eV}$ indicating it is predominately in the unoxidised $\mathrm{S}^{2+}$ form $(-\mathrm{S}-,-\mathrm{S}-\mathrm{S}-)$, Fig. 1 . The smaller peak intensity at $168.0 \mathrm{eV}$ suggests that re-oxidation of the alkali reduced sulphur dye thiols to reform the insoluble disulphide bond-based sulphur dye has resulted in over-oxidation to $S^{6+}$ species. The $S^{6+}$ oxidised dye derivatives may be assigned to $-\mathrm{SO}_{3}^{-},-\mathrm{S}-\mathrm{SO}_{2}-$ or $-\mathrm{S}-\mathrm{SO}_{3}^{-}$species and are most likely the precursors to loss of dye from the fibre. Interestingly there is little spectral intensity at $166.0 \mathrm{eV}$ which could be attributed to any intermediate oxidised $S^{4+}$ species.

The effect of the ISO 105 CO6 washing with perborate was to increase the amount of higher binding energy spectral component at $168.0 \mathrm{eV}$, Fig. 2, indicating an increase in the oxidised $\mathrm{S}^{6+}$ dye species. This oxidised sulphur species spectral intensity was further increased with repeated ISO 105 CO6 washings with perborate,

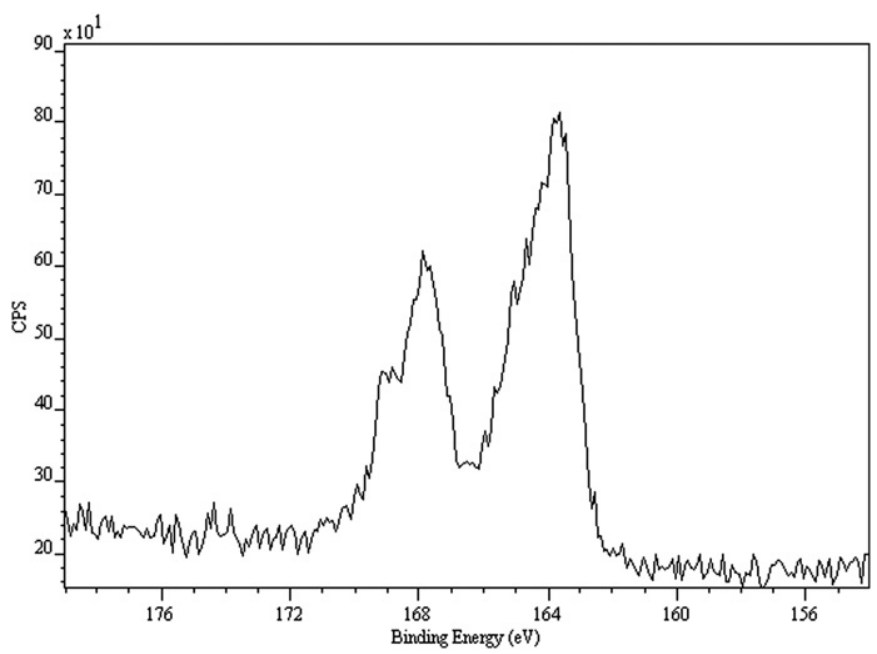

Fig. 2. $S(2 p) X P$ spectrum of sulphur black dyed cotton laundered with sodium perborate and stainless steel balls (ISO 105 CO6).

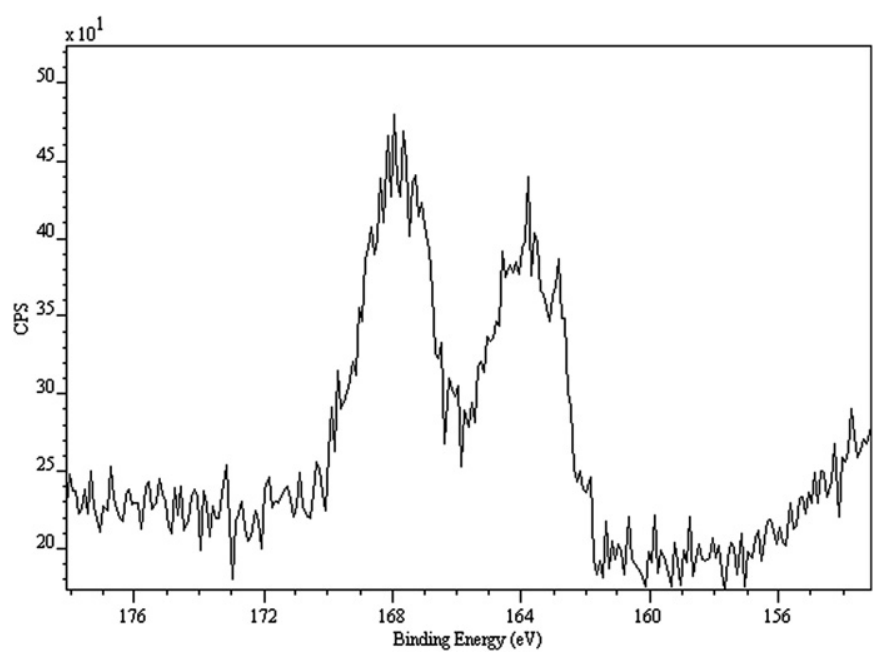

Fig. 3. $S(2 p) X P$ spectrum of sulphur black dyed cotton laundered with sodium perborate and stainless steel balls (ISO 105 CO6 - triplicate wash cycles).

Fig. 3. The XP spectral difference between perborate-based washing with or without the stainless steel balls was minimal in terms of any increased surface oxidation. It can therefore be concluded that the addition of steel balls into the washing procedure does not directly affect the formation of oxidised dye sulphur species at the fibre surface.

The effect of individual and repeated ISO 105 CO6 washing of the sulphur dyed cotton without perborate was also to increase the level of oxidised sulphur at $168.0 \mathrm{eV}$ in the $\mathrm{S}(2 \mathrm{p}) \mathrm{XP}$ spectra, Figs. 4 and 5 . However the oxidative effect was not as great as that observed with the perborate-based launderings. In particular the loss of sulphur from the fibre surface is minimal in the case of the non-perborate based laundering regimes, while it is significant in the presence of perborate (3.2\% and 1.6\% Atomic Sulphur, respectively, Table 1) It is presumed that oxidation of the dye disulphide bonds leads to the formation of water-soluble sulphonated short chain dye fragments which are lost into solution.

\subsection{ISO 105 CO9 washing}

Examination of the S(2p) XP spectrum of the ISO 105 CO9 washed C. I. Sulphur Black 1 dyed cotton fabric demonstrated that

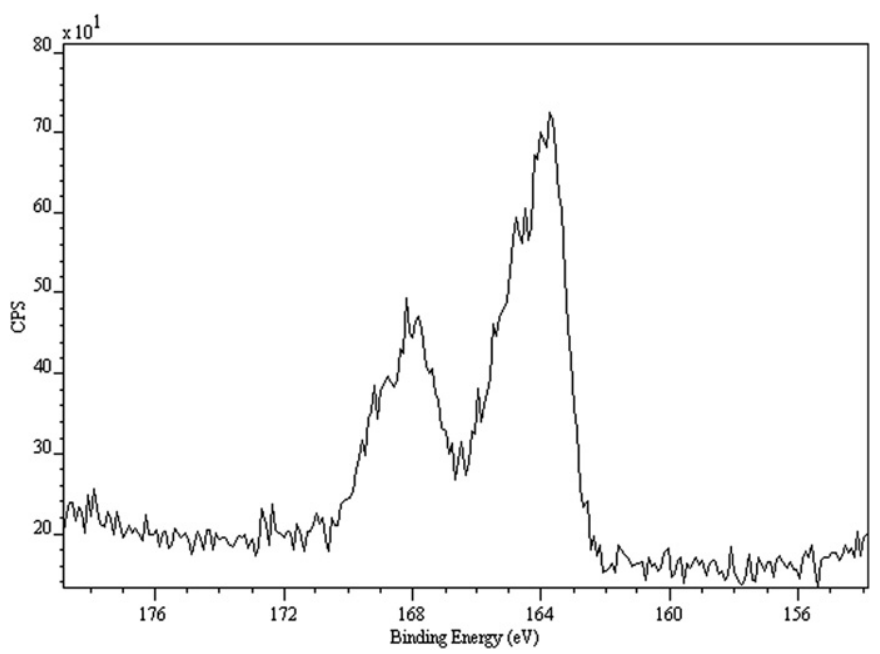

Fig. 4. $S(2 p) X P$ spectrum of sulphur black dyed cotton laundered without sodium perborate and with stainless steel balls (ISO 105 CO6). 


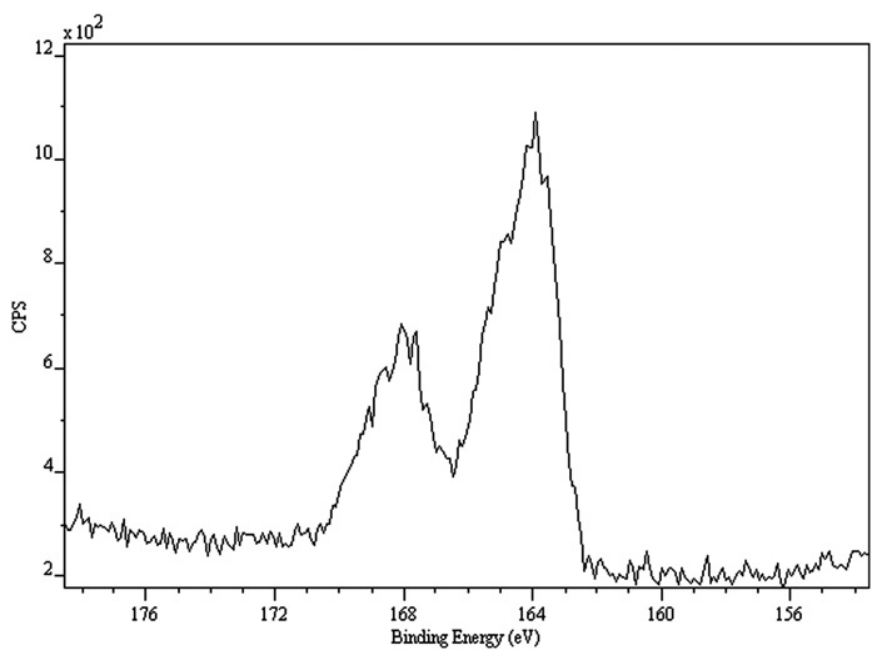

Fig. 5. $S(2 p) X P$ spectrum of sulphur black dyed cotton laundered without sodium perborate and without stainless balls (ISO 105 CO6 - triplicate wash cycles).

the effect of the much more aggressive TAED catalysed perborate treatment was to convert most of the surface sulphur to the oxidised $\mathrm{S}^{6+}$ form, Fig. 6 , in addition significant concomitant reduction of the surface sulphur concentration (from $3.0 \%$ to $0.6 \%$ Atomic Sulphur, Table 1) probably due to loss of the sulphonated dye fragments into solution was observed. In contrast the $S(2 p) X P$ spectrum of the ISO 105 CO9 washed without perborate fabric, Fig. 7, mirrored the low level of oxidation and minimal loss of sulphur observed for the comparable ISO 105 CO6, without perborate, washed cotton fabric, Fig. 4.

Examination of the $\mathrm{C}(1 \mathrm{~s}) \mathrm{XP}$ spectrum of C. I. Sulphur Black 1 dyed cotton fabric demonstrates the presence non-cellulosic carbon species at the fibre surface, Fig. 8; specifically the major peak intensity at $285.0 \mathrm{eV}$ assigned to $\mathrm{C}-\mathrm{C}$ and $\mathrm{C}-\mathrm{H}$ species. If the fibre surface were purely cellulosic the expected carbon spectral peak intensities would occur at 286.6 and $288.0 \mathrm{eV}$, with an intensity ratio of 5:1 respectively, and could be assigned to:

$$
\begin{aligned}
& 286.6 \text { eV } \mathrm{C}-\mathrm{OH} \\
& 288.0 \text { eV } \mathrm{O}-\underline{\mathrm{C}}-\mathrm{O}
\end{aligned}
$$

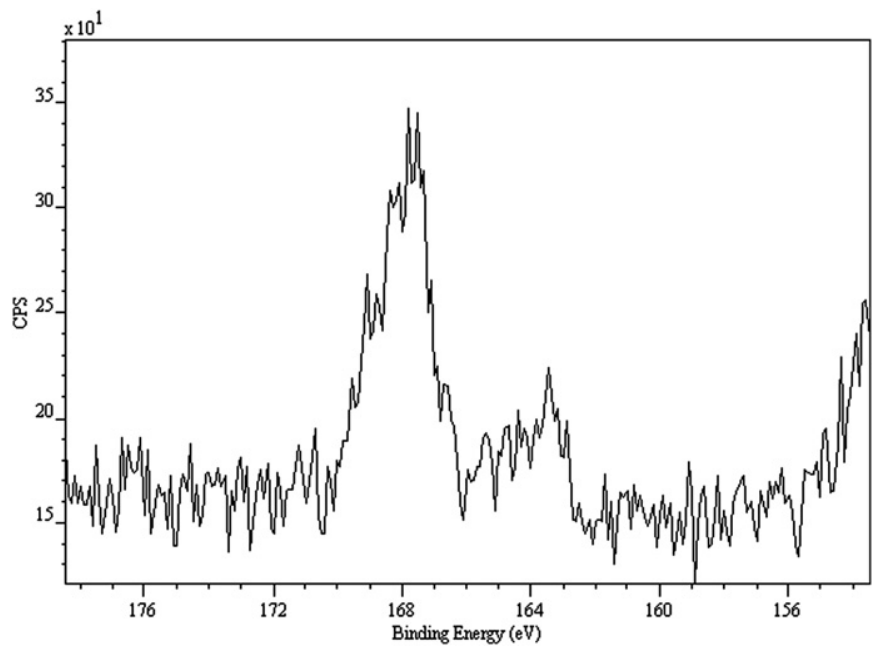

Fig. 6. $S(2 p) X P$ spectrum of sulphur black dyed cotton laundered with sodium perborate and stainless steel balls (ISO 105 CO9).

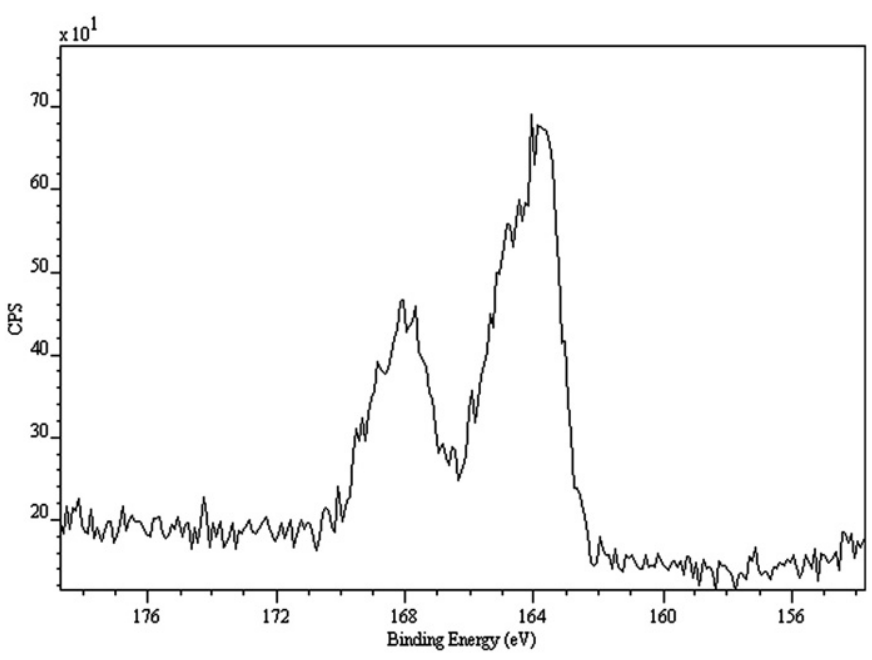

Fig. 7. $\mathrm{S}(2 \mathrm{p}) \mathrm{XP}$ spectrum of sulphur black dyed cotton laundered without sodium perborate and with stainless steel balls (ISO 105 CO9).

However evidence of oxidised functionalities at the fibre surface is also observed; these other $\mathrm{C}(1 \mathrm{~s})$ species can be attributed to:

$$
\begin{aligned}
& 288.8 \mathrm{eV} \mathrm{C}=\mathrm{O} \\
& 289.0 \mathrm{eV} \overline{\mathrm{O}}=\underline{\mathrm{C}}-\mathrm{OH}
\end{aligned}
$$

The effect of the ISO 105 CO9 laundering with perborate on $\mathrm{C}(1 \mathrm{~s})$ spectral intensities was to reduce the hydrocarbon signal intensity at $285.0 \mathrm{eV}$ and to increase the oxidised species intensities at 286.6, 288.0 and $289.0 \mathrm{eV}$, Fig. 9. The surface carbon, nitrogen and sulphur content also decreased while the surface oxygen content clearly increased indicating exposure of the cotton to a highly oxidative environment, Table 1 . The effect of comparable laundering without perborate present was also to reduce the hydrocarbon signal intensity at $285.0 \mathrm{eV}$ but to a much lesser extent, Fig. 10, reflecting the far less oxidative and degradative environment.

\subsection{Influence of ISO 105 CO6/9 washing on fabric colour}

The effect of ISO 105 CO6 washing with and without perborate was to marginally reduce the colour strength and increase fabric

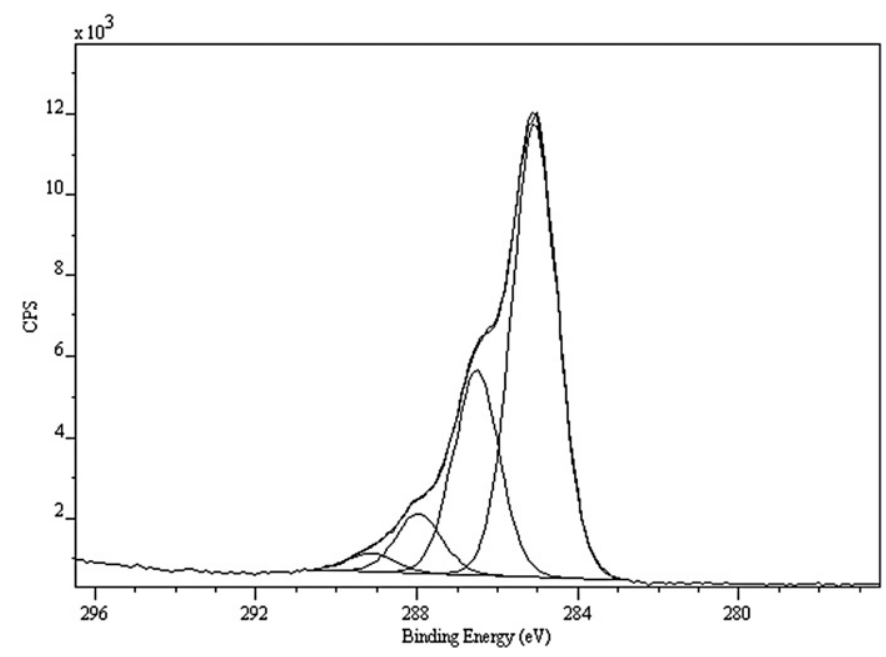

Fig. 8. $C(1 \mathrm{~s}) \mathrm{XP}$ spectrum of unlaundered sulphur black dyed cotton fabric. 


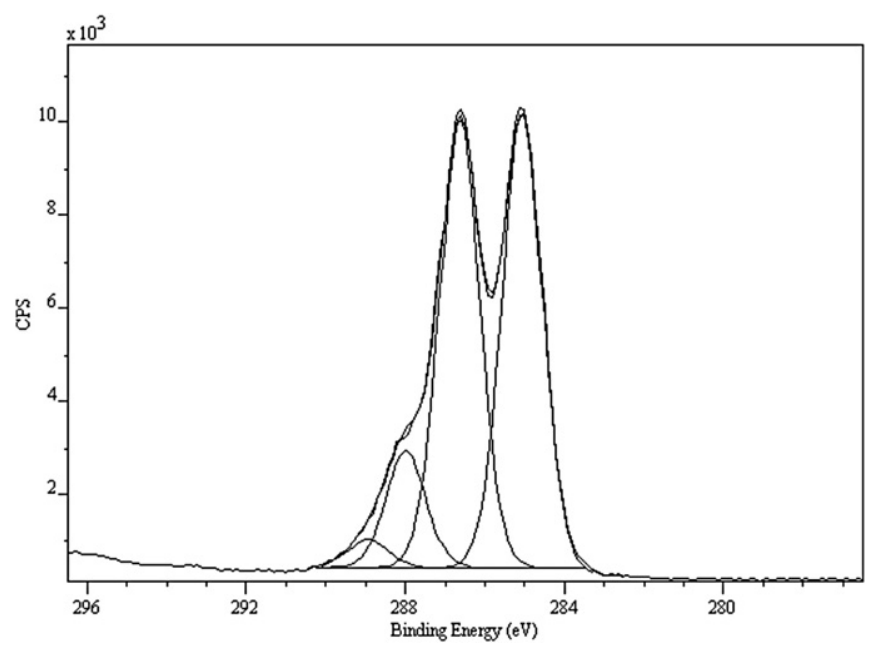

Fig. 9. $C(1 \mathrm{~s}) \mathrm{XP}$ spectrum of sulphur black dyed cotton laundered with sodium perborate and stainless steel balls (ISO 105 CO9).

lightness, Table 2. With repeated (triplicate) ISO 105 CO6 washing with perborate however the fabric colour strength clearly decreased and the fabric lightness increased. These results are in good agreement with the XPS analysis data where increased oxidation of dye sulphur $S^{2+}$ to $S^{6+}$ species and the concomitant decrease in surface atomic \% sulphur content were observed. In contrast, the fabric subjected to triplicate ISO 105 CO6 washing without perborate the colour and lightness change was greater than the single washed fabric, but as expected this change was however far smaller in magnitude than that observed with the fabric that had been triplicate washed with the oxidative perborate solution.

For the single ISO 105 CO9 washing with perborate, a much larger colour strength and lightness change was observed (K/S and $L^{*}$ values in Table 2 ) probably due to the catalysed perborate/TAED bleaching of the dyed fabric causing oxidation of the sulphur dye to water soluble $S^{6+}$ dye fragments. In contrast the ISO 105 CO9 washing without perborate showed a similar decrease in colour strength to that observed to the ISO 105 CO6 washing without perborate procedure.

The effect of introducing stainless steel balls into the washing process was to increase abrasion of the fibre/fabric surface. This process may remove surface dye and potentially increase fibrillation of the fibres. The fibrillation of cellulosic fibres can be an

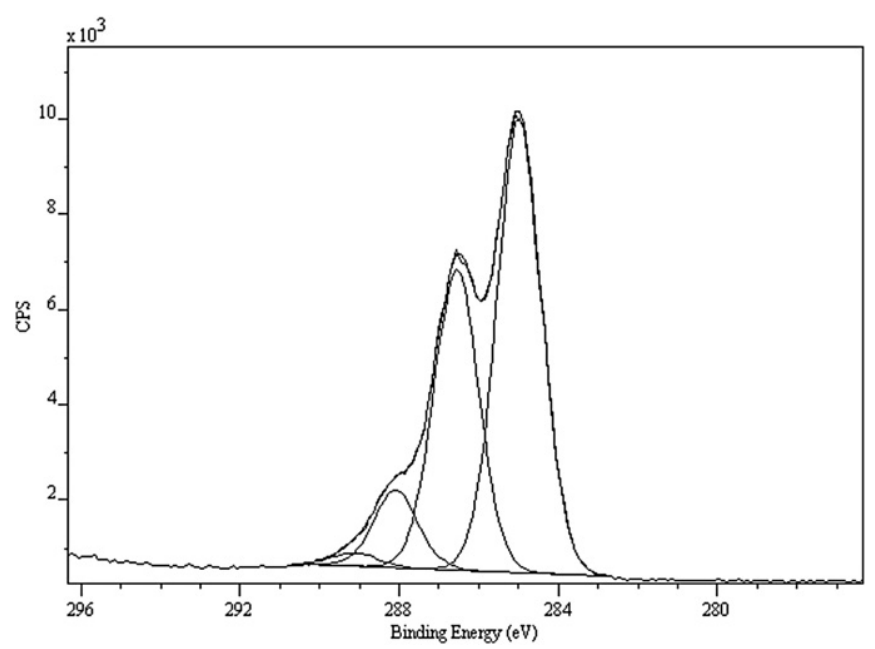

Fig. 10. $C(1 \mathrm{~s}) \mathrm{XP}$ spectrum of sulphur black dyed cotton laundered without sodium perborate and with stainless steel balls (ISO 105 CO9). important physical process in the fading of dyed textiles where the specular light reflectance at smooth fibre surfaces is replaced by diffuse reflectance resulting in an apparent loss in colour strength and an increase in fabric lightness [16-21]. Examination of the ISO 105 CO6 washing treatments of the dyed fabrics with and without the abrasive stainless steel balls indicates that the balls tended to further increase the fabric lightness and decrease the colour strength, Table 2, while XPS analysis suggests little additional dye is lost through the addition of steel balls in the washing process, Table 1.

Scanning Electron Microscopic analysis of the fabrics washed with the steel balls indicates there is significantly more fibrillation of the cotton fibres. Coupled with associated dye loss through washing, the fibrillation at the fibre surface is the likely cause of increased lightness and colour strength loss.

\section{Conclusions}

The surface sensitive XPS technique has demonstrated the presence of the sulphur-based colourant within the outer 3-10 nm of the fibre surface of $C$. I. Sulphur Black 1 dyed cotton fabric. The sulphur atoms attributed to the surface sulphur dye are predominantly in the $S^{2+}$ form, occurring as $-S-$ or $-S-S-$, with a lesser amount of oxidised $\mathrm{S}^{6+}$ possibly occurring as $-\mathrm{SO}_{3}^{-},-\mathrm{S}-\mathrm{SO}_{2}-$ or $-\mathrm{S}-\mathrm{SO}_{3}^{-}$species. These oxidised surface species have probably arisen due to over-oxidation of the dye during the dyeing process.

The effect of ISO 105 CO6 washing with perborate was to increase the higher oxidation state $\mathrm{S}(2 \mathrm{p})$ signal intensity at $168 \mathrm{eV}$ and the concomitant visual loss in colour, as indicated by decreased colour strength $K / S$ and increased lightness $L^{*}$ values. The effect of ISO 105 CO6 washing without perborate was also to increase the higher oxidation state $\mathrm{S}(2 \mathrm{p})$ signal intensity at $168 \mathrm{eV}$ but to a significantly smaller extent. The effect of including stainless steel balls in the washing test was to increase surface fibrillation and increase the fabric lightness by introducing greater diffuse reflectance at the fibre/fabric surface.

Laundering the sulphur dyed cotton using the ISO 105 CO9 protocol resulted in far greater surface oxidation, surface dye loss and colour change due to the activated bleaching environment. There was no evidence for the formation of $\mathrm{S}^{4+}$ dye species at the fibre surface but it is probable that dye loss occurs through conversion of the disulphide bonds in the sulphur dye to watersolubilising sulphonate species and the subsequent loss of short chain dye fragments into solution.

\section{Acknowledgements}

This research was funded, in part, through a PhD scholarship provided by the Textile Division of the National Research Center, Egypt. The authors thank Phil Cohen for his help in dyeing the cotton fabric and in undertaking the fastness testing. In addition the authors thank Alison Harvey and Dr. John Walton for their help in the XPS surface study and Dr. Huw Owens in the colour analysis.

\section{References}

[1] Shore J. Cellulosic dyeing. Bradford: Society of Dyers and Colourist; 1995. p. 280-313.

[2] Broadbent AD. Basic principles of textile colouration. Bradford: Society of Dyers and Colourist; 2001. p. 379-87.

[3] Czajkowski W, Misztal J. The use of thiourea dioxide as reducing agent in the application of sulphur dyes. Dyes and Pigments 1994;26:77-81.

[4] Burkinshaw SM, Collins JW. Aftertreatments to improve the wash fastness of sulphur dyeings on cotton. Dyes and Pigments 1995;29:323-34.

[5] Blackburn RS, Harvey A. Green chemistry methods in sulphur dyeing: application of various reducing D-sugars and analysis of the importance of optimum redox. Environmental Science \& Technology 2004;38:4034-9. 
[6] Burkinshaw SM, Gotsopoulos A. The pre-treatment of cotton to enhance its dyeability. I. Sulphur dyes. Dyes and Pigments 1996;32:209-28.

[7] Bozic M, Kokol V. Ecological alternatives to the reduction and oxidation processes in dyeing with vat and sulphur dyes. Dyes and Pigments 2008;76: 299-309.

[8] Ibrahim NA, El Gamal AR, Mahrous F. Improving the environmental aspects of sulphur dyeing of cotton knitted fabrics. Journal of Natural Fibres 2008;5: 238-50.

[9] Wang W, Zhang S, Yang J. A water soluble cationic sulphur dye for the dyeing of silk. Coloration Technology 2005;121:245-8.

[10] BOL. ISO 105 CO6 C1S/C2STextiles - tests for colour fastness. Colour fastness to domestic and commercial laundering [accessed November 2010], https://bsol bsigroup.com/en/Bsol-Item-Detail-Page/?pid=000000000030193640; 2010.

[11] Phillips D, Bevan G, Lloyd J, Hall R, Hoffmeister J. The colour fastness of dyed fabrics to activated oxygen bleach-containing detergent: an international interlaboratory trial. JSDC 1999;115:100-1.

[12] Phillips D, Scotney J, Percival R, Bevan G, Lloyd J. Colour fastness to industrial laundering: an international inter-laboratory trial of the proposed ISO 105 C12 test procedure. Coloration Technology 2002;118:277-80.

[13] BSI. ISO 105 CO9Textiles - tests for colour fastness. Colour fastness to domestic and commercial laundering. Oxidative bleach response using a nonphosphate reference detergent incorporating a low temperature bleach activator [accessed November 2010], https://bsol.bsigroup.com/en/Bsol-ItemDetail-Page/?pid=000000000030169957; 2003.

[14] Ratner B, Castner D. In: Vickerman JC, Gilmore IS, editors. Electron spectroscopy for chemical analysis in surface analysis - the principal techniques. Chichester: John Wiley and Sons Ltd;; 2009. p. 47-112.

[15] Mitchell R, Carr C, Parfitt M, Vickerman J, Jones C. Surface chemical analysis of raw cotton fibres and associated materials. Cellulose 2005;12:629-39.

[16] Nicolai M, Nechwatal A, Mieck KP. Lyocell-fasern-alternativen zum reduzieren der fibrillierung. Mell Textil 1999;79:848-51.

[17] Cavaco-Paulo A, Morgado J. Indigo backstaining during cellulase washing. Textile Research Journal 1998;68:398-401 [20] Hohberg T, Thumm S. Veredlung von lyocell. Mell Textil 1998; 79: 253-256.

[18] Nicolai M, Nechwatal A, Mieck KP. Textile crosslinking reactions to reduce the fibrillation tendency of lyocell fibers. Textile Research Journal 1996;66: 575-80.

[19] Nechwatal A, Nicolai M, Mieck KP, Heublein B, Kuhne G, Klemm D. Studies on the wet fibrillation of lyocell. Angew Makromol Chem 1999;271:84-92.

[20] Rosunee S, Carr CM, Wilding MA. Effect of aqueous temperature on the yarnon-yarn abrasion of tencel. Adv Col Sci \& Tech 2001;4:28-32.

[21] Diresul sulphur dyes manual: S. A. Cardoner Ltd. Spain.

[22] Walton J, Wincott P, Fairley N, Carrick A. Peak fitting with Casa XPS, a Casa pocket book. Cheshire: Acolyte Science; 2010. p. 5-45, 111-116. 